\title{
Retrograde degeneration of retinal ganglion cells in homonymous hemianopsia
}

\author{
This article was published in the following Dove Press journal: \\ Clinical Ophthalmology \\ II June 2015 \\ Number of times this article has been viewed
}

\section{Angela M Herro \\ Byron L Lam}

Bascom Palmer Eye Institute, Department of Ophthalmology, University of Miami Miller School of Medicine, Miami, FL, USA
Correspondence: Angela M Herro Horizon Eye Specialists and Lasik Center, I8325 N. Allied Way, Suite 100, Phoenix, AZ 85054, USA

Tel + I 602790226 I

Fax +| 480419540 |

Email angela.herro@gmail.com
Background: The aim of this study was to demonstrate the relationship between topographic reduction in macular ganglion cell complex (GCC) thickness as detected with spectral-domain optical coherence tomography and visual field defects caused by ischemic occipital cortical injury.

Methods: This study was a retrospective review of all patients who presented to our eye institution between January 2012 and July 2014 with visual field defects secondary to ischemic cortical injury. The visual field defect pattern and mean deviation were analyzed. Retinal nerve fiber layer (RNFL) and macular GCC were both assessed with spectral-domain optical coherence tomography. Patients with any ocular pathology that could affect these measurements were excluded. The topographic relationship of visual field defect to reduction in GCC was specifically analyzed.

Results: Nine patients met the inclusion criteria. Their average age was 65 (57-73) years; eight were men and six had right hemianopsias. The laterality of the visual field defect was used to assign an affected and unaffected side of analysis for RNFL and GCC layer thickness. A right hemianopsia meant that the nasal fibers of the right eye and temporal fibers of the left eye were assigned as the "affected side", and the temporal fibers of the right eye and nasal fibers of the left eye were assigned as "unaffected". There was no statistically significant difference between affected and unaffected RNFL. However, there was a significant difference in GCC layer reduction between the affected and unaffected sides $(P=0.029)$.

Conclusion: There is evidence of retrograde trans-synaptic retinal ganglion cell loss in patients with homonymous hemianopsias from cortical visual impairment. This relationship is reflected in thinning of the GCC and maintains the topographic relationship of the visual field defect.

Keywords: homonymous hemianopsia, ganglion cell complex, occipital lobe

\section{Introduction}

Axonal degeneration is a phenomenon of the central and peripheral nervous system wherein a nerve fiber will lose function after an injury. This process begins at the site of injury, and can happen in an anterograde (Wallerian) fashion, toward the post-synaptic end, or in a retrograde fashion (toward the presynaptic cell body). This process of degeneration applies to the injured nerve body and axon, and can occur as early as 5-30 minutes after a traumatic event. ${ }^{1}$ When the degeneration crosses a synapse, it is referred to as trans-synaptic, and this can also proceed in an anterograde or posterograde mechanism. Anterograde transneuronal degeneration occurs secondary to loss of excitatory input to the post-synaptic neuron. Retrograde transneuronal damage is theorized to be caused by a loss of trophic support from the injured cell to the presynaptic dendritic tree.

Examples of neuronal degeneration can be seen in the human visual pathway. In glaucoma, the site of pathology or injury is the optic nerve head. Retrograde 
degeneration can be seen by thinning of the retinal nerve fiber layer (RNFL) and, more recently, the ganglion cell complex (GCC) of the retina. ${ }^{2}$ Anterograde degeneration in glaucoma can be seen by optic nerve atrophy on neuroimaging, as well as evidence of trans-synaptic damage by demonstrating morphologic changes in the visual processing cortex ${ }^{3}$ and loss of cell density in the lateral geniculate nucleus of macaque monkeys after enucleation. ${ }^{4,5}$ However, there is little evidence of retrograde transneuronal degeneration in the human visual pathway. It is traditionally believed that there are no clinical signs of optic nerve damage after occipital lobe damage acquired in adulthood. This belief has already been challenged over recent years with studies demonstrating optic disc pallor, loss of retinal cells, and a relative afferent pupillary defect in post-geniculate pathology. ${ }^{6-8}$ More recently, other investigators have attempted to quantitatively measure this effect by RNFL and GCC analysis using spectral-domain optical coherence tomography (SD-OCT). ${ }^{9}$

In this study, analysis of both the RNFL and the GCC were included to demonstrate retrograde transneuronal degeneration in patients with homonymous hemianopsia caused by cerebrovascular accident (CVA). Prior to the algorithm for GCC measurement, the best way to indirectly measure the nerve fiber layer was to use the RNFL function on OCT. The RNFL is primarily reported divided into four quadrants, ie, temporal, nasal, superior, and inferior. This distinction is less than ideal for evaluating post-chiasmatic visual field defects since the temporal quadrant contains retinal fibers from the temporal retina as well as the maculopapillary bundle nasal to the fovea. This allows for both the nasal and temporal visual fields to be represented in the same measurement, and could be a confounder when attempting to analyze visual field defects that respect the midline. To demonstrate retrograde transsynaptic degeneration, a right occipital lesion would cause thinning of the nasal nerve fiber of the right eye and temporal nerve fiber of the left eye. This becomes further challenging as the peripapillary nerve fiber layer is not symmetric at baseline in normal subjects. In general, the inferior quadrant is thickest, followed by the superior, nasal, and temporal.

The fovea represents the physiologic division between crossed and uncrossed fibers to the visual cortex, and at baseline is symmetric between the superior, inferior, nasal, and temporal quadrants. For these reasons, the GCC analysis is a more straightforward way to demonstrate thinning as it is projected to the visual cortex and measured by a visual field. The GCC has been shown to be a more sensitive marker of inner retinal thinning than the RNFL in a number of disease processes. ${ }^{10-13}$ This has allowed clinicians to diagnose and treat earlier to prevent further visual disability.

\section{Materials and methods}

All patients who presented to the Bascom Palmer Eye Institute with documented homonymous hemianopsia on Humphrey visual field (HVF) testing due to ischemic stroke between January 2012 and July 2014 were retrospectively reviewed. This study was approved and conformed to the policies and procedures outlined by the institutional review board of the University of Miami and conformed to the requirements of the United States Health Insurance Portability and Privacy Act. Case identification was done through a search using the International Classification of Diseases, 9th Revision for the term "homonymous hemianopsia". Additional inclusion criteria consisted of required SD-OCT of the RNFL and macula with GCC analysis, and documentation of a cortical stroke on magnetic resonance imaging consistent with the visual field defect. The HVF protocol was a Swedish Interactive Test Algorithm (SITA) standard 30-2 algorithm. All patients with any other ocular comorbidities that could produce a visual field defect or reduction of GCC or RNFL were excluded. Specifically excluded were patients with glaucoma, other known optic neuropathy, or age-related macular degeneration. Patients with hypertension, hypercholesterolemia, or diabetes were not excluded because these systemic diagnoses are also risk factors for stroke, and thus most patients had at least one of these conditions. However, patients with background diabetic retinopathy or hypertensive retinopathy were not included, as this has been found to produce changes in the nerve fiber layer. ${ }^{14}$ Demographics recorded included age, sex, laterality, date of ischemic event, and date of ophthalmic examination.

Visual fields included for analysis were those with parameters of fixation losses, along with false positives and negatives of $<25 \%$, consistent with reliability indices in the glaucomatous population, ${ }^{15}$ and the analysis was performed using the averaged mean deviation (MD) of the affected hemifield compared with the averaged MD of the unaffected hemifield. The average $\mathrm{MD}$ of the affected hemifield of all eyes were used as datum points for the affected group and the average MD of unaffected hemifields were used for the unaffected group (Figure 1).

OCT scans were included if the signal strength was $\geq 7$ without blink artifact. The RNFL analysis was divided into two components, ie, primarily crossed and primarily uncrossed fibers. The RNFL is reported in four quadrants which are not symmetric at baseline, so the nasal and temporal fibers cannot be directly compared. Further, the temporal RNFL includes fibers from the nasal and temporal visual field, so can cause confusion when relating this to hemianopsias. Because of this, the quadrants were not compared between an individual eye but between fellow eyes. Nasal fibers were chosen to represent crossed fibers and the average of the remaining temporal, superior, and 
Central 30-2 threshold test

Fixation monitor: gaze/blind spot Fixation target: central Fixation losses: 4/16 False POS errors: $0 \%$ False NEG errors: $0 \%$ Test duration: 07:17

Fovea: $37 \mathrm{~dB}$

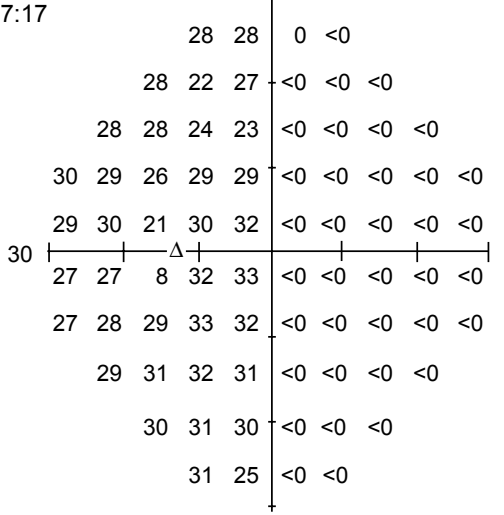

\section{Pupil diameter: $5.7 \mathrm{~mm} \quad$ Date: 04-01-2014 Visual acuity: RX: +5.00 DS DC X Age: 57}

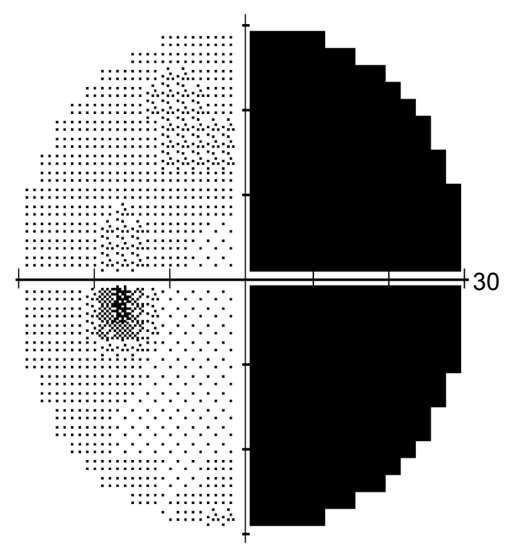

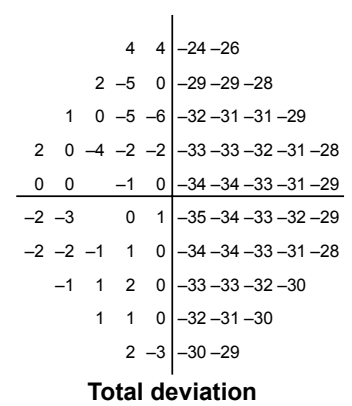

Total deviation
Strategy: SITA-sta

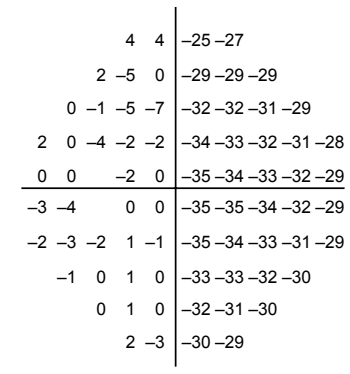

Pattern deviation

Central 30-2 threshold test

Fixation monitor: gaze/blind spot Fixation target: central Fixation losses: 0/16 False POS errors: $3 \%$ False NEG errors: $0 \%$ Test duration: 07:09

Fovea: $37 \mathrm{~dB}$
Stimulus: III, white Background: 31.5 ASB Strategy: SITA-standard

\section{Pupil diameter: $6.0 \mathrm{~mm}$ Date: 04-01-2014 Visual acuity: RX: +5.25 DS DC X Age: 57}

Outside normal limits

VFI $\quad 47 \%$

MD $\quad-17.85 \mathrm{~dB} P<0.5 \%$

PSD $18.68 \mathrm{~dB} P<0.5 \%$

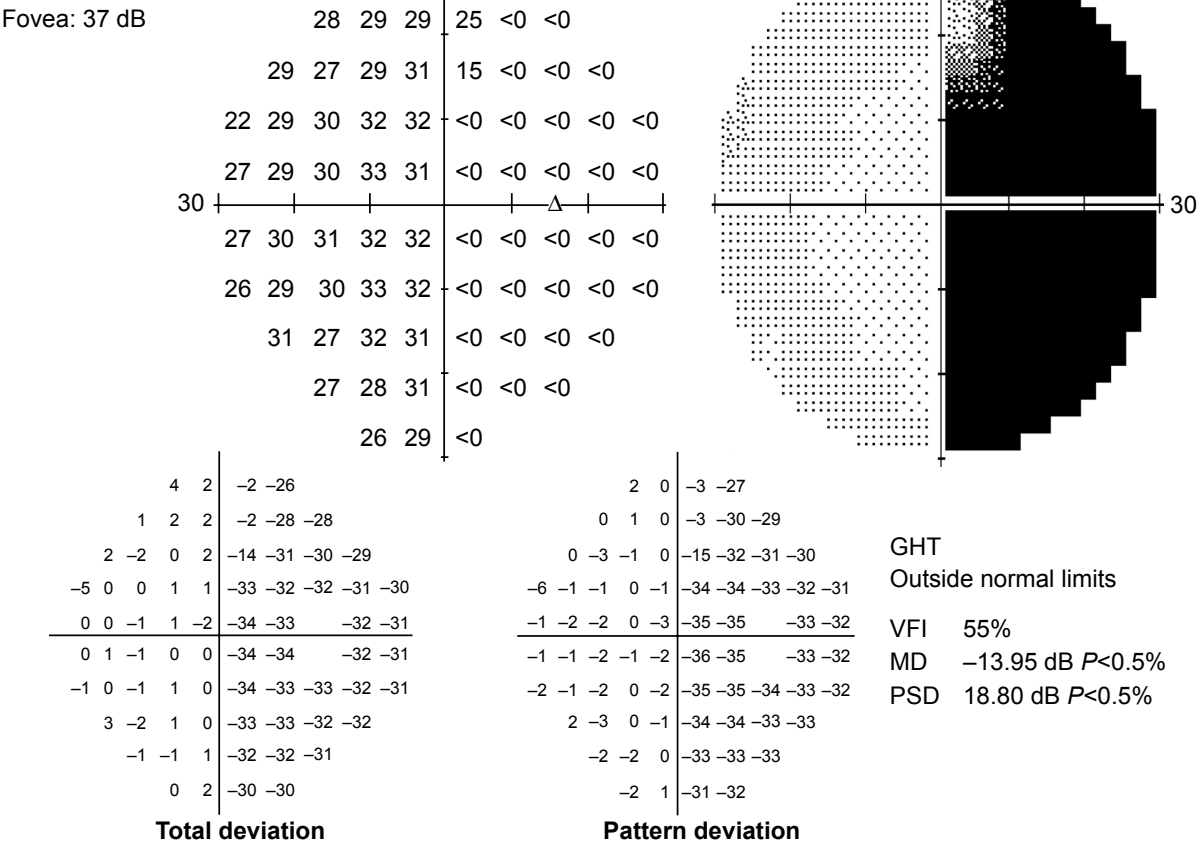

Figure I Humphrey visual field for patient I showing gray scale image and total and pattern deviation demonstrating dense right homonymous hemianopsia. Abbreviations: MD, Mean deviation; PSD, Pattern standard deviation. 
inferior fibers was chosen to represent the uncrossed fibers. The baseline variation in RNFL thickness amongst quadrants was accounted for by only comparing similar quadrants, ie, nasal affected versus nasal unaffected in the fellow eye (Figure 2A). The analysis involved a Student's $t$-test comparing all affected nasal quadrant measurements as one group compared with all unaffected nasal quadrant measurements. Another Student's $t$-test was performed similarly comparing an average of the remaining three quadrants, ie, temporal, superior, and inferior. The thickness of each RNFL quadrant was also recorded as green (within the 95th percentile), yellow (bottom 5 th percentile), or red (bottom 1st percentile).

The GCC analysis differed from the RNFL in that the GCC was compared using the affected and unaffected hemiretina, so each eye has a pair of datum points. For example, for patients with a right homonymous hemianopsia, the temporal hemiretina of the left eye was labeled affected and the nasal hemiretina as unaffected. The GCC algorithm on SD-OCT (Cirrus HD-OCT, Carl Zeiss Meditec, Dublin, CA, USA) divides the macula into six equal sectors across a

A

Signal strength: $10 / 10 \quad 10 / 10$

\begin{tabular}{|c|c|c|c|c|}
\hline \multicolumn{5}{|c|}{ ONH and RNFL OU analysis: optic disc cube $200 \times 200$} \\
\hline \multirow{3}{*}{350} & RNFL thickness map & $\bigwedge$ & OD & os \\
\hline & & Average RNFL thickness & $117 \mu \mathrm{m}$ & $105 \mu \mathrm{m}$ \\
\hline & & RNFL symmetry & \multicolumn{2}{|c|}{$73 \%$} \\
\hline \multirow{4}{*}{175} & & Rim area & $1.19 \mathrm{~mm}^{2}$ & $1.15 \mathrm{~mm}^{2}$ \\
\hline & & Disc area & $1.88 \mathrm{~mm}^{2}$ & $2.00 \mathrm{~mm}^{2}$ \\
\hline & & Average $\mathrm{C} / \mathrm{D}$ ratio & 0.60 & 0.66 \\
\hline & & Vertical $C / D$ ratio & 0.58 & 0.64 \\
\hline$\Delta I$ & & Cup volume & $0.266 \mathrm{~mm}^{3}$ & $0.536 \mathrm{~mm}^{3}$ \\
\hline
\end{tabular}
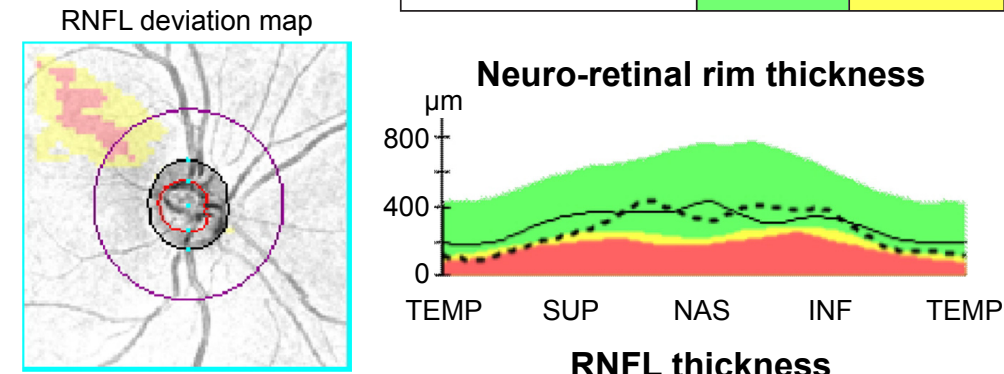

Disc center $(0.00,0.06) \mathrm{mm}$

Extracted horizontal tomogram
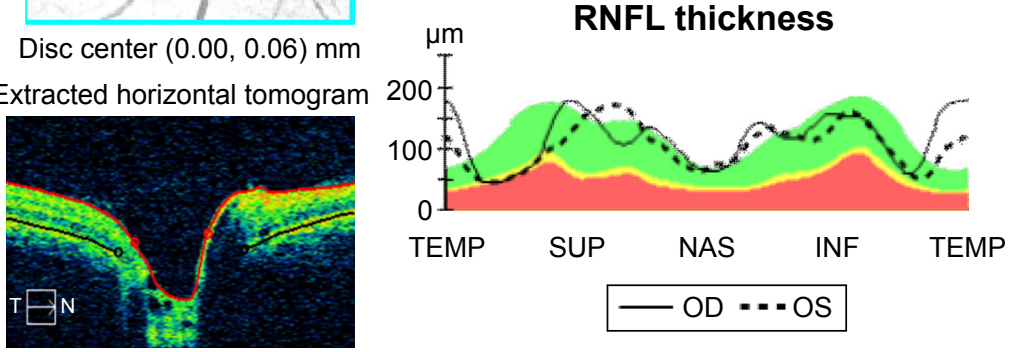

Extracted vertical tomogram
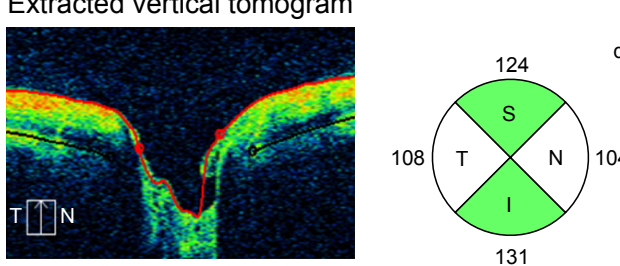

Diversified:

distribution of normals

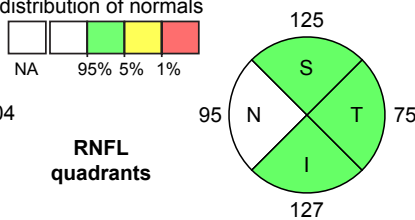

RNFL circular tomogram
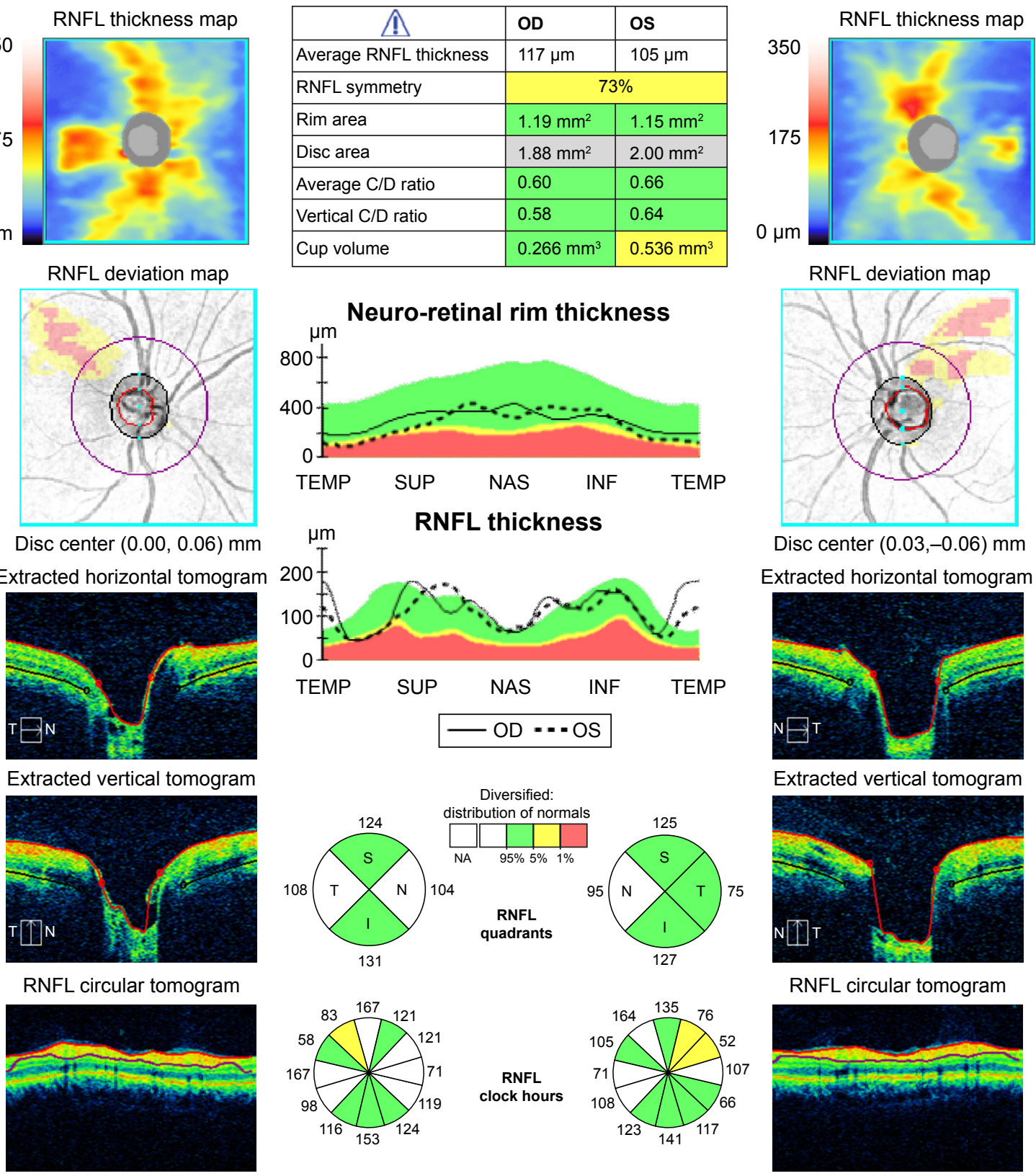

RNFL deviation map

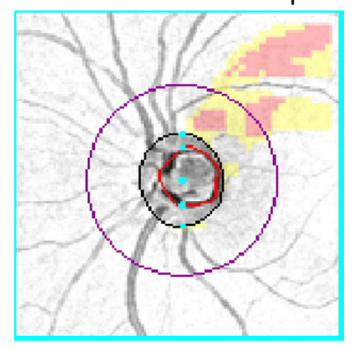

Disc center $(0.03,-0.06) \mathrm{mm}$

Extracted horizontal tomogram

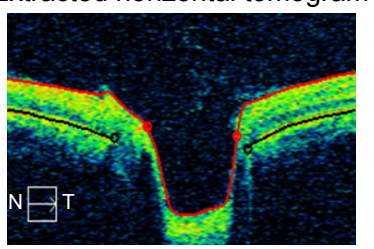

Extracted vertical tomogram

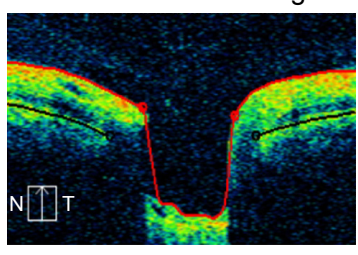

RNFL circular tomogram

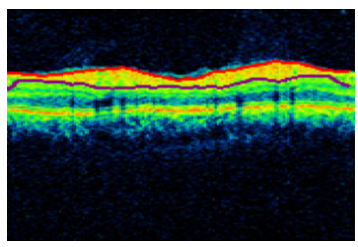




\section{B} Signal strength: $9 / 10$

$10 / 10$

\section{Ganglion cell OU analysis: macular cube $200 \times 200$}

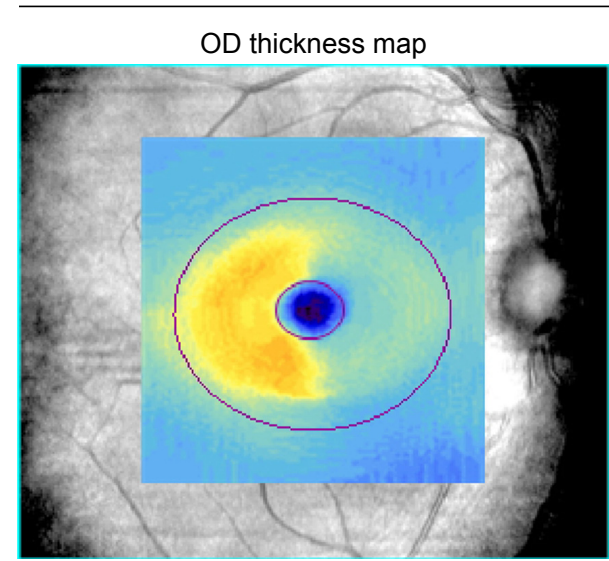

Fovea: 99, 101

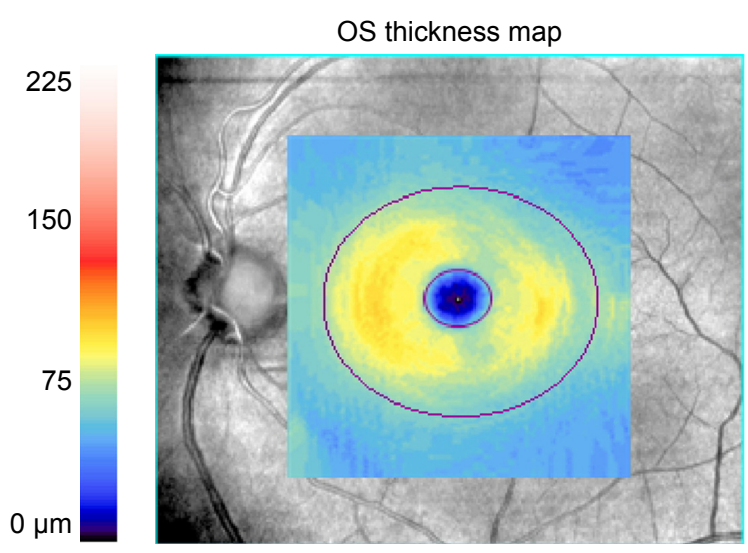

Fovea: 100, 96
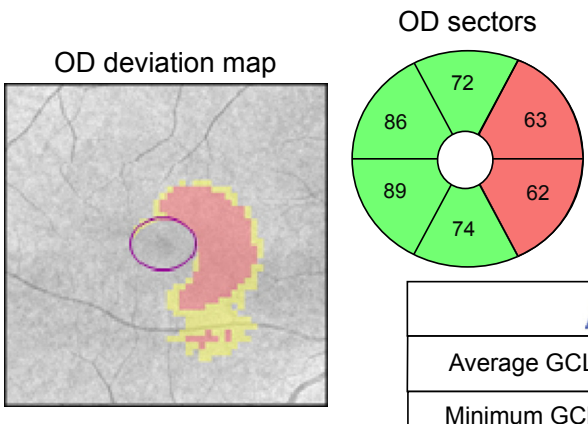

Diversified: distribution of normals

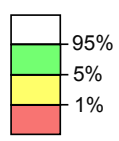

\begin{tabular}{|l|l|l|}
\hline \multicolumn{1}{|c|}{} & OD $\boldsymbol{\mu m}$ & OS $\boldsymbol{\mu m}$ \\
\hline Average GCL + IPL thickness & 74 & 75 \\
\hline Minimum GCL + IPL thickness & 60 & 67 \\
\hline
\end{tabular}

OS sectors

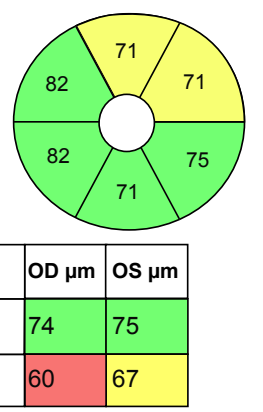

OS deviation map

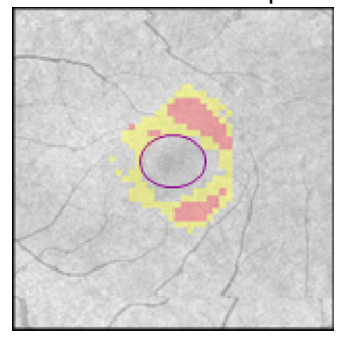

OD horizontal B-scan

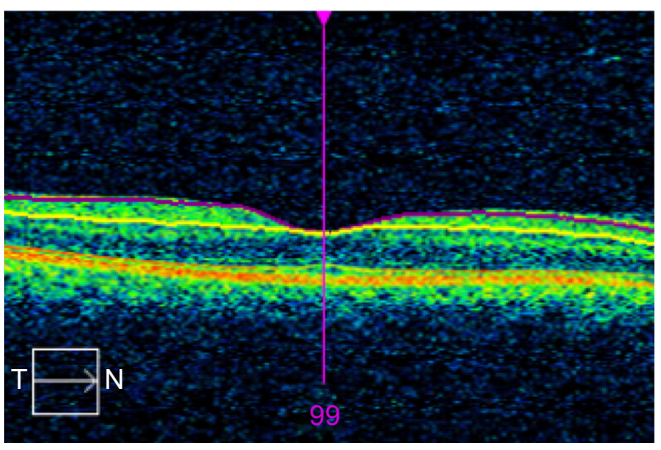

OS horizontal B-scan

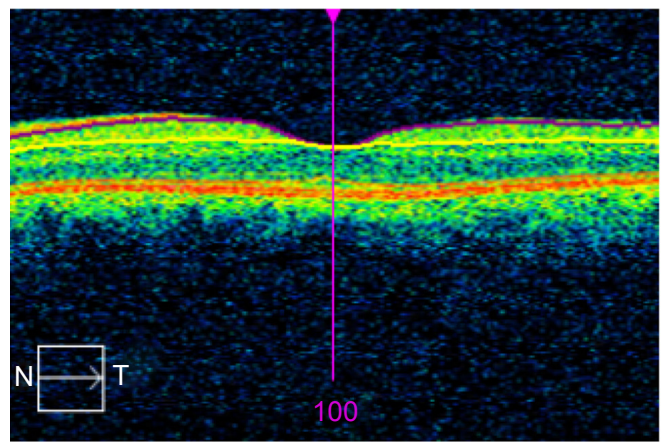

Figure 2 (A) SD-OCT RNFL for patient I showing normal thickness without a relative difference in thickness between affected and unaffected fibers as well as an example of how the calculations were performed. (B) SD-OCT ganglion cell complex for patient I showing relative thinning in the nasal macula of the right eye and temporal macula of the left eye corresponding to the right hemianopsia.

Abbreviations: RNFL, retinal nerve fiber layer; SD-OCT, spectral-domain optical coherence tomography; IPL, inner plexiform layer; GCL, ganglion cell layer; ONH, optic nerve head; OD, right eye; OS, left eye; OU, both eyes.

horizontal meridian. At baseline, all segments should be of equal thickness. To establish a thickness measurement for the nasal macula, the superonasal sector and the inferonasal sector were averaged, and similarly the temporal thickness was calculated as the average of the superotemporal and inferotemporal sectors. The affected hemiretinal measurements were compared against a group of all unaffected sectors (Figure 2B). The inferior and superior segments were left out of the analysis as there is no vertical midline to keep the crossed and uncrossed fibers separated.
The statistical analysis was performed using Statistical Package for the Social Sciences version 22 (SPSS Inc, Chicago, IL, USA). Since visual field data follows a normal distribution, a matched Student's $t$-test was used to compare the mean deviation of affected and unaffected quadrants $(\mathrm{n}=34)$. The RNFL and GCC analyses were obtained using Wilcoxon signed-rank tests because of the lack of normal distribution and small sample size. The HVF MD and GCC analysis compared affected versus unaffected hemifields/ hemiretinas while the RNFL analysis compared nasal fibers 
between unaffected and affected eyes and combined temporal, superior, and inferior fibers between unaffected and affected eyes separately. $P<0.05$ was deemed to be statistically significant. Where the sample size precluded calculation of a $P$-value, a critical value of $\mathrm{W}$ was calculated using the Wilcoxon signed-rank test, and statistical significance was reported based on this value.

\section{Results}

Nine patients (17 eyes) met the inclusion criteria for this study. One eye of one patient was excluded secondary to ophthalmic artery occlusion. This patient was excluded from RNFL analysis; however, his unaffected eye was included in the visual field and GCC analyses. The baseline demographics and clinical data are presented in Table 1. All patients were over the age of 50 years at the time of inclusion, with an average age of 65 years. The HVF MD between affected and unaffected hemifields was $-26.5 \mathrm{~dB}$ and $-7.3 \mathrm{~dB}$, respectively $(P<0.0001)$. The average RNFL thickness was $74.1 \mu \mathrm{m}$ for affected nasal fibers and $74.1 \mu \mathrm{m}$ for unaffected nasal fibers. The average thickness of the combined temporal, superior, and inferior fibers was $90.63 \mu \mathrm{m}$ for the affected sectors and $88.6 \mu \mathrm{m}$ for the combined unaffected sectors. These results were not found to be significant because the W-value was above the calculated critical value. The RNFL quadrants were all within the 95 th percentile for thickness in $67 \%$ of patients. The remaining $33 \%$ of patients had thinning in the bottom 1 st or 5 th percentiles that did not consistently follow a pattern related to their visual field loss. The GCC analysis revealed an average thickness of $69.9 \mu \mathrm{m}$ in the affected hemiretina and $75.2 \mu \mathrm{m}$ in the unaffected hemiretina $(P=0.029)$.

A representative case (patient 1 ) is a 57-year-old man who presented to the neuro-ophthalmology clinic for evaluation of a visual field defect found on routine ophthalmologic testing. His best-corrected visual acuity was 20/20 in the right eye and 20/25 in the left eye. Confrontation visual field testing revealed a dense right homonymous hemianopsia. The remainder of the clinical examination, including pupillary assessment, intraocular pressure, and external slit-lamp and funduscopic evaluation, was within normal limits. Magnetic resonance imaging was obtained that demonstrated an old left occipital infarction with encephalomalacia (Figure 3). Automated HVF 30-2 testing corroborated the confrontation fields, with an MD of $-29.6 \mathrm{~dB}$ for the right hemifield of the right eye and $-31.2 \mathrm{~dB}$ for the right hemifield in the left eye, with an MD of $1.15 \mathrm{~dB}$ and $1.5 \mathrm{~dB}$ in the respective left hemifields (Figure 1). The RNFL showed an average affected nasal thickness of $104 \mu \mathrm{m}$ in the affected nasal quadrant and $95 \mu \mathrm{m}$ in the unaffected nasal quadrant. The combined temporal/superior/inferior value was $120 \mu \mathrm{m}$ for the unaffected sectors compared with $109 \mu \mathrm{m}$ for the affected sectors (Figure 2A). The GCC demonstrated generalized thinning that was more prominent for the hemisphere corresponding to the visual field defect (Figure $2 \mathrm{~B}$ ). The right eye demonstrated an average thickness of $87.5 \mu \mathrm{m}$ in the temporal unaffected sectors compared with $62.5 \mu \mathrm{m}$ in the nasal affected sectors. Similarly for the left eye, $82 \mu \mathrm{m}$ versus $73 \mu \mathrm{m}$ in the unaffected and affected sectors, respectively.

Table I Patient demographics and clinical data

\begin{tabular}{|c|c|c|c|c|c|c|c|c|c|c|c|c|}
\hline \multirow[t]{2}{*}{$\begin{array}{l}\text { Patient } \\
\text { Number }\end{array}$} & \multirow[t]{2}{*}{$\begin{array}{l}\text { Age } \\
\text { (years) }\end{array}$} & \multirow[t]{2}{*}{ Sex } & \multirow[t]{2}{*}{ Side } & \multirow[t]{2}{*}{$\begin{array}{l}\text { Time from CVA } \\
\text { to examination }\end{array}$} & \multicolumn{2}{|c|}{$\begin{array}{l}\text { HVF } 30-2 \\
\text { (MD, dB) }\end{array}$} & \multicolumn{2}{|c|}{$\begin{array}{l}\text { RNFL OD } \\
(\mu \mathrm{m})\end{array}$} & \multicolumn{2}{|c|}{$\begin{array}{l}\text { RNFL OS } \\
(\mu \mathrm{m})\end{array}$} & \multicolumn{2}{|c|}{ GCC $(\mu \mathrm{m})$} \\
\hline & & & & & A & $\mathbf{U}$ & $\mathbf{A}$ & $\mathbf{U}$ & $\mathbf{A}$ & $\mathbf{U}$ & $\mathbf{A}$ & $\mathbf{U}$ \\
\hline 1 & 57 & M & $\mathrm{R}$ & Unknown & -30.4 & -1.3 & 104 & 121 & 109 & 95 & 67.8 & 84.8 \\
\hline 2 & 72 & $\mathrm{~F}$ & $\mathrm{R}$ & Unknown & -25.3 & -1.5 & 52 & 80.3 & 86.7 & 54 & 59.5 & 74.8 \\
\hline 3 & 65 & M & $\mathrm{L}$ & 3 months & -21.9 & -1.8 & 67 & 81 & 86.7 & 69 & 75.2 & 74.8 \\
\hline 4 & 60 & M & $\mathrm{R}$ & Unknown & -29.4 & -25.5 & 67 & 104 & 103 & 69 & 77 & 76.3 \\
\hline 5 & 60 & M & $\mathrm{L}$ & Unknown & -25.4 & -8.1 & 73 & 106 & 98 & 73 & 74.7 & 79 \\
\hline 6 & 58 & $M$ & $\mathrm{R}$ & Unknown & -30.4 & -2.1 & 64 & 103 & 102.7 & 57 & 81 & 80 \\
\hline 7 & 72 & $M$ & $\mathrm{R}$ & $>14$ months & -25.5 & -2.4 & 57 & 71.7 & 62 & 57 & 63 & 72 \\
\hline $8^{*}$ & 73 & $M$ & $\mathrm{R}$ & Unknown & -22.7 & -17.6 & - & - & - & - & 67 & 68 \\
\hline 9 & 70 & $M$ & $\mathrm{~L}$ & Unknown & -27.3 & -5.1 & 92.3 & 76 & 74 & 91 & 64 & 68 \\
\hline Mean & 65.2 & & & & -26.5 & -7.3 & & & & & 69.9 & 75.2 \\
\hline SD & 6.61 & & & & 6.10 & 8.46 & & & & & 7.98 & 6.68 \\
\hline$P$-value & & & & & $<0.001$ & & & & & & 0.029 & \\
\hline
\end{tabular}

Notes: *This patient's right eye was excluded secondary to ophthalmic artery occlusion that led to diffuse RNFL and GCC thinning. The GCC measurements listed are for his left eye only; the affected visual field category is the average of the affected hemifield in the right and left eyes; there are two separate RNFL calculations, ie, nasal affected versus nasal unaffected: mean $74.1 \pm 19.49 \mu \mathrm{m}$ versus $74 . \mathrm{I} \pm 18.96 \mu \mathrm{m} ; P=0.99$; and combined $\mathrm{T} / \mathrm{S} / \mathrm{I}$ affected versus unaffected: $90.63 \pm \mathrm{I} 8.0 \mathrm{I} \mu \mathrm{m}$ versus $88.6 \pm \mathrm{I} 8.8 \mathrm{I} \mu \mathrm{m}$; the $\mathrm{GCC}$ reported in the affected column is the average of the affected hemiretina in the right and left eyes.

Abbreviations: CVA, cerebrovascular accident; GCC, ganglion cell complex; HVF, Humphrey visual field; A, affected; U, unaffected; MD, mean deviation; T/S/I, combined temporal, superior, inferior; SD, standard deviation; RNFL, retinal nerve fiber layer. 


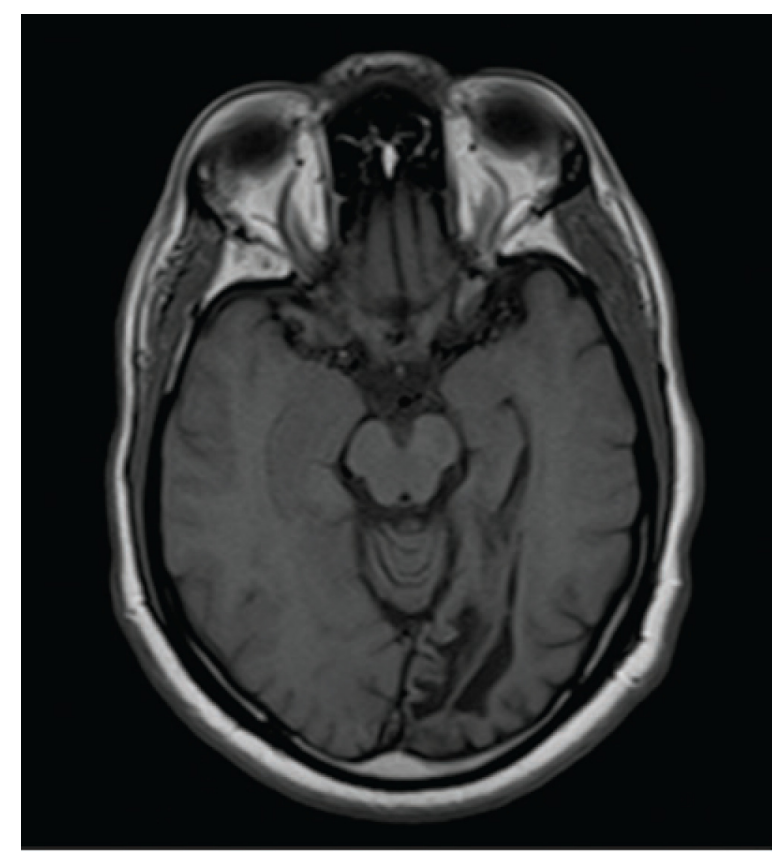

Figure 3 Axial TI-weighted magnetic resonance image of patient I demonstrating encephalomalacia of the left occipital lobe.

Magnetic resonance imaging was ordered, and revealed left occipital encephalomalacia consistent with an old ischemic infarction, explaining the visual field defect.

\section{Discussion}

The theories that cortical injuries do not produce measurable optic nerve damage and that retrograde trans-synaptic neuronal degeneration does not occur have been challenged for over 40 years. More recently, evidence has shown a reduction in RNFL thickness in patients with CVAs of the anterior or posterior circulation as compared with normal controls. ${ }^{16}$ This study demonstrates thinning of the retinal ganglion cell layer after cortical visual impairment. This was measured using patients with homonymous hemianopsia and compared the laterality of stroke with the corresponding hemiretina.

The RNFL results demonstrate generalized thinning of the RNFL in 33\% of patients using the standard deviations of the computer-based algorithm of the OCT. These results are lower than previously published data on RNFL thinning after cerebral infarction. This may be due to lack of information regarding time from CVA to examination. It is possible that the patients without thinning were imaged within 2 years of CVA, making it less likely to demonstrate a pattern reduction of the RNFL. ${ }^{9} 16,17$ There was no difference between affected and unaffected sectors of the RNFL; however, the study is limited by the small number of patients. Most of the patients in the study had normal RNFL thickness in all quadrants, and therefore would be unlikely to demonstrate a difference between affected and unaffected fibers. The retrospective nature of the study did not allow us to determine the time from CVA to examination in most patients. Further studies with follow-up and known time of CVA are needed. Jindahra et al reported more specifically on an identifiable time course from CVA to RNFL thinning, and showed that there is more rapid thinning in the first 2 years, with continued loss of thickness at a lower rate thereafter. ${ }^{17,18}$ Other studies have shown a decrease in the RNFL and inner retina in patients with various neurologic diseases of the retrogeniculate pathway, as well as specifically pointing out GCC thinning as evidence of trans-synaptic retrograde degeneration. ${ }^{9} 10,16$ With increased use of this technology and more use of OCT to detect subtle macular changes related to vision loss or effects of medication, new patterns have been increasingly observed.

However, there are few studies assessing macular GCC thickness as it relates to visual field defects from cortical lesions. Here, the effects of cortical damage in ganglion cells by trans-synaptic retrograde degeneration is specifically addressed in documenting and analyzing the GCC. GCC analysis is a more specific measure of inner retinal thinning because it is an indirect measure of both the inner plexiform layer and the ganglion cell layers of the retina. It is centered over the fovea, ie, the physiologic divide for crossed and uncrossed fibers to the visual cortex, as opposed to the optic nerve in the RNFL algorithm. This makes it unique in evaluating for retinal sequelae of post-geniculate pathology, specifically hemianopsia from ischemic stroke. In normal individuals, GCC thickness is seen as a symmetric ring centered on the fovea, and the pattern of thinning can be readily appreciated. This is a simple, noninvasive test that does not rely on patient responses to produce quantifiable measures of GCC thinning. The GCC in this study was found to be significantly reduced in the affected hemiretina as compared with the unaffected hemiretina. This supports the theory that retrograde neuronal degeneration can occur in the visual pathway and maintains the topographic distribution of the ganglion cell layer as projected to the visual cortex.

The GCC is measured using OCT, which is a simple, noninvasive test that does not rely on patient responses to produce quantifiable measures of GCC thinning. This is important clinically as an adjunct to visual field testing to confirm a visual field defect in a patient who is unable to undergo a reliable visual field test or may have other neurologic or ophthalmic comorbidities. This is valuable information for family members, caregivers, and rehabilitation teams, so that they can orient treatment to the unaffected side, as well as for clinicians to be aware if a new pattern has emerged on GCC testing that was previously indeterminate on visual field and 
funduscopic examinations. Monitoring the GCC over time may allow prediction of the timing of the ischemic event if it is unknown, as well as aid in mapping the time course of trans-synaptic degeneration. More studies with larger sample sizes and a prospective design are necessary to elucidate these factors and allow for better diagnosis and treatment.

The limitations of this study include its small sample size and retrospective design. There were no age-matched normal controls to compare GCC thickness on the unaffected side or to compare RNFL thickness. The authors attempted to exclude patients with pathology that could affect the unaffected hemiretina; however, because the data analysis was paired, each patient acted as their own control. The analysis was geared toward determining the difference between the affected and unaffected fibers as they are projected to the visual cortex, so if there was generalized reduction of GCC, it should not have significantly affected the results. Another limitation is the lack of information regarding the time course of degeneration. This is due to the fact that many patients are unaware of a visual field defect and do not seek prompt medical attention. Future studies should be conducted in a prospective manner utilizing primary care, emergency medicine, and neurology as important referral sources to identify these patients as early as possible.

\section{Conclusion}

Occipital ischemia can cause retrograde trans-synaptic axonal degeneration that maintains the topographic relationship of the retina as projected to the visual cortex. This can be demonstrated by the relationship between GCC reduction on OCT and visual field defects in homonymous hemianopsia. This study should act more as a proof of theory that this relationship exists and is measurable. Larger prospective studies are necessary to define an association and time course more clearly.

\section{Acknowledgments}

This research was funded in part by a grant from the Department of Defense (W81XWH-09-1-0674) and by Research to Prevent Blindness Inc.

\section{Disclosure}

The authors report no conflicts of interest in this work.

\section{References}

1. Wang JT, Medress ZA, Barres BA. Axon degeneration: molecular mechanisms of a self-destruction pathway. J Cell Biol. 2012;196:7-18.

2. Medeiros FA, Zangwill LM, Alencar LM, et al. Detection of glaucoma progression with stratus OCT retinal nerve fiber layer, optic nerve head, and macular thickness measurements. Invest Ophthalmol Vis Sci. 2009; 50:5741-5748.

3. Yu L, Yin X, Dai C, et al. Morphologic changes in the anterior and posterior subregions of $\mathrm{V} 1$ and $\mathrm{V} 2$ and the V5/MT+ in patients with primary open angle glaucoma. Brain Res. 2014;1588:135-143.

4. Matthews MR. Further observations on transneuronal degeneration in the lateral geniculate nucleus of the macaque monkey. J Anat. 1964;98: 255-263.

5. Goldby F. A note on transneuronal atrophy in the human lateral geniculate body. J Neurol Neurosurg Psychiatry. 1957;20:202-207.

6. Cowey A, Alexander I, Stoerig P. Transneuronal retrograde degeneration of retinal ganglion cells and optic tract in hemianopic monkeys and humans. Brain. 2011;134:2149-2157.

7. Papageorgiou E, Ticini LF, Hardiess G, et al. The pupillary light reflex pathway: cytoarchitectonic probabilistic maps in hemianopic patients. Neurology. 2008;70:956-963.

8. Vanburen JM. Trans-synaptic retrograde degeneration in the visual system of primates. J Neurol Neurosurg Psychiatry. 1963;26:402-409.

9. Jindahra P, Hedges TR, Mendoza Santiesteban CE, et al. Optical coherence tomography of the retina: applications in neurology. Curr Opin Neurol. 2010;23:16-23.

10. Yamashita T, Miki A, Iguchi Y, et al. Reduced retinal ganglion cell complex thickness in patients with posterior cerebral artery infarction detected using spectral-domain optical coherence tomography. Jpn $J$ Ophthalmol. 2012;56:502-510.

11. Balk LJ, Steenwijk MD, Tewarie P, et al. Bidirectional trans-synaptic axonal degeneration in the visual pathway in multiple sclerosis. J Neurol Neurosurg Psychiatry. 2014;86:419-424.

12. Kim MJ, Park KH, Yoo BW, et al. Comparison of macular GCIPL and peripapillary RNFL deviation maps for detection of glaucomatous eye localized RNFL defect. Acta Ophthalmol. 2015;93:e22-e28.

13. Padhy D, Rao A. Macular ganglion cell/inner plexiform layer measurements by spectral-domain optical coherence tomography for detection of early glaucoma and comparison to retinal nerve fiber layer measurements. Am J Ophthalmol. 2014;158:211.

14. Park HY, Kim IT, Park CK. Early diabetic changes in the nerve fiber layer at the macula detected by spectral-domain optical coherence tomography. Br J Ophthalmol. 2011;95:1223-1228.

15. Birt CM, Shin DH, Samudrala V, et al. Analysis of reliability indices from Humphrey visual field tests in an urban glaucoma population. Ophthalmology. 1997;104:1126-11230.

16. Park HY, Park YG, Cho AH, et al. Transneuronal retrograde degeneration of the retinal ganglion cells in patients with cerebral infarction. Ophthalmology. 2013;120:1292-1299.

17. Jindahra P, Petrie A, Plant GT. Retrograde trans-synaptic retinal ganglion cell loss identified by optical coherence tomography. Brain. 2009; 132:628-634.

18. Jindahra P, Petrie A, Plant GT. The time course of retrograde transsynaptic degeneration following occipital lobe damage in humans. Brain. 2012;135:534-541.
Clinical Ophthalmology

\section{Publish your work in this journal}

Clinical Ophthalmology is an international, peer-reviewed journal covering all subspecialties within ophthalmology. Key topics include: Optometry; Visual science; Pharmacology and drug therapy in eye diseases; Basic Sciences; Primary and Secondary eye care; Patient Safety and Quality of Care Improvements. This journal is indexed on Submit your manuscript here: http://www.dovepress.com/clinical-ophthalmology-journal

\section{Dovepress}

PubMed Central and CAS, and is the official journal of The Society of Clinical Ophthalmology (SCO). The manuscript management system is completely online and includes a very quick and fair peer-review system, which is all easy to use. Visit http://www.dovepress.com/ testimonials.php to read real quotes from published authors. 